\title{
EARLY VERSUS LATE ONSET ATTENTION DEFICIT DISORDER
}

The validity of the age-of-onset criterion (AOC), with symptoms emerging before age 7 , in the diagnosis of attention deficit/hyperactivity disorder was evaluated by analysis of data from interviews with 9- to 16-year-olds from the Great Smoky Mountains Study, at the University of North Carolina, Chapel Hill, NC. A majority of youths with ADHD were reported to first exhibit symptoms in early childhood and before age 7 . Most parents considered symptoms to have "always" been present, without a specific date of onset. The percentage of youths first exhibiting symptoms after age 7 was higher in the inattentive group (26\%) than in the hyperactive-impulsive $(8 \%)$ and combined groups (13\%). Patients with early onset inattentive symptoms were at increased risk for comorbid ODD, while those with late-onset inattentive symptoms were at increased risk for comorbid depression. The current AOC results in the underidentification of youths in the inattentive group, late onset type. The continued inclusion of the AOC for the assessment of combined but not the inattentive type of ADHD is supported. Early onset ADHD was associated with worse clinical outcome in youths with the combined subtype but not with the inattentive subtype. (Willoughby MT, Curran PJ, Costello EJ, Angold A. Implications of early versus late onset of attention deficity/hyperactivity disorder symptoms. I Am Acad Child Adolesc Psychiatry December 2000;39:1512-1519). (Reprints: Mr Willoughby, Davie Hall CB \#3270, Department of Psychology, University of North Carolina, Chapel Hill, NC 27599).

COMMENT. The recent challenge to the inclusion of an age-of-onset criterion for the diagnosis of ADHD may be valid for patients with the inattentive subtype. The current AOC of less than 7 years may exclude patients in the late onset inattentive group, but it appears to be valid for the assessment of the combined group.

\section{METHYLPHENIDATE TOXIC EXPOSURE REPORTS}

Methylphenidate (MPH) exposures reported to a regional poison control center during a 2-year period were analysed at Wayne State University School of Medicine, Detroit, MI. Of 289 patients exposed, $41 \%$ had taken their own medication. Only cases involving non-sustained release MPH were included. The mean overdose of MPH ingested was $1.7 \mathrm{mg} / \mathrm{kg}$ (range, 0.06-29 mg/kg). Symptoms, most commonly tachycardia, agitation, and lethargy, developed in 31\%; and showed a dose-response relationship. Symptoms were more severe and 3 times as frequent with intentional compared to unintentional exposures. Of 149 accidental ingestions, 143 were in children, of whom $65 \%$ developed symptoms, including tachycardia, agitation, insomnia and rash. Multiple symptoms including abdominal pain, emesis, or hypertension, in combination with tachycardia or agitation, occurred in the remainder. Most therapeutic errors occurred in the 6 to 11 year-old group, whereas the highest rate of symptoms was reported in the 0 to 5 year-old group. These included lethargy, agitation, headache, dystonia, and vomiting. Outcome was not significantly altered by gastric decontamination, performed in 105 patients. The peak age period for exposure was 6 to 9 years, and therapeutic error was the most common reason. (White SR, Yadao CM. Characterization of methylphenidate exposures reported to a regional poison control center. Arch Pediatr Adolesc Med Dec 2000;154:1199-1203). (Reprints: Suzanne R White MD, Children's Hospital of Michigan Regional Poison Control Center, Suite 616, 4160 John R, Detroit, MI 48201).

COMMENT. Methylphenidate exposure is associated with symptoms in one 